\title{
Propuesta de un formulario abierto para un programa de atención médica dirigida en México
}

\author{
Comité Terapéutico y Farmacológico de México*
}

\section{Comité Terapéutico y Farmacológico de México. Propuesta de un formulario abierto para un programa de atención médica dirigida en México. Salud Publica Mex 1999;41:27-41.}

\begin{abstract}
Resumen
Objetivo. Integrar un formulario abierto con recomendaciones para el uso de medicamentos en la práctica médica privada, útil para que el médico encuentre un espectro terapéutico amplio que le permita seleccionar la mejor alternativa para su paciente. Material y métodos Un comité médico interdisciplinario de alto nivel científico revisó todos y cada uno de los ingredientes activos comercializados y disponibles para su venta en México; esta revisión formó parte de un programa de administración de beneficios farmacéuticos y atención médica dirigida en nuestro medio. El comité clasificó cada uno de los medicamentos de acuerdo con las siguientes catego rías: a) esenciales; b) excluidos, y c) no esenciales-no excluidos. Los criterios de dicha clasificación se definieron a partir de un estricto análisis de medicina, basado en evidencia y en el marco de un riguroso sustento científico. Resultados. El formulario propuesto está integrado por un total de 1106 ingredientes activos - solos o combinados-, de los cuales 429 (38.8\%) se catalogaron como esenciales. Cerca de uno de cada 10 fármacos (8.7\%) utilizados en la actualidad en la práctica médica privada en México se clasificaron como excluidos, por aspectos relacionados con su eficacia o su seguridad. Algunos de estos medicamentos son altamente prescritos. Conclusiones En este trabajo se detalla el proceso que se siguió para integrar el comite que clasificó los medicamentos, sus objetivos y los criterios establecidos para su funcionamiento, y se exponen las bases para estructurar la lista final de ingredientes activos que conforman el formulario abierto que aquí se propone.
\end{abstract}

Palabras clave: economía farmacéutica; formularios; administración de beneficios farmacéuticos; atención médica dirigida; México

\author{
Comité Terapéutico y Farmacológico de México. \\ Open formulary propossal \\ for a managed care program \\ in Mexico. \\ Salud Publica Mex 1999;41:27-41.
}

\begin{abstract}
A bstract
Objective. To integrate an open formulary with drug use recommendations in which a physician could find a wide range of therapeutic options for his private practice. Material and methods. An interdisciplinary medical committee with a high scientific level, reviewed each one of all the drugs available to be sell in Mexico, as part of a Pharmacy Benefit Management \& Manage Care program implemented in our country. Each drug was classified according to its active ingredient under one of the following catego ries: 1) essentials, 2) excluded, and 3) not essentials - not excluded The decision in each case was based on pure scientific grounds and using an Evidence-Based Medicine analysis. ResultsThe proposed formulary contains a to tal of 1106 active ingredients -either unique or in combination-, from which $429(38.8 \%)$ were classified as essentials. About one-out-of-ten drugs (8.7\%) currently in use within the private medical practice in Mexico, some of them highly prescribed, were regarded by the committee as excluded due to concerns about their efficacy and/or or safety. Conclusions $0 \mathrm{n}$ this report a detailed description of the process followed to conform the committee, its objectives an its performing criteria is presented, as well as the bases under which the final list of drugs of the formulary were set.
\end{abstract}

Key words: economics, pharmaceutical; formularies; pharmacy benefit management; managed care; Mexico

* Integrantes del Comité Terapéutico y Farmacológico de México: Dr. José Luis Arredondo-García (pediatría/infectología), D r. Augusto Bondani-G uasti (farmacología química), Dr. Rubén Burgos-Vargas (reumatología), D r. Ernesto Castelazo-Morales (ginecología y obstetricia), Dr. Jaime de la GarzaSalazar (oncología), Dr. Carlos Jerjes-Sánchez-Días (cardiología/neumología), Dr. David Kershenobich-Stalnikowitz (gastroenterología), Dr. Alberto Lifshitz-G uinzberg (medicina interna), D r. Eduardo Zorrilla-Hernández (endocrinología), D r. José Luis Viramontes-Madrid (coordinador).

Fecha de recibido: 22 de abril de 1998 • Fecha de aprobado: 10 de diciembre de 1998

Solicitud de sobretiros: Dr. José Luis Viramontes Madrid. Medco de México Managed Care, S. de R. L. de C.V. Av. Insurgentes Sur 800, piso 12, colonia del Valle, 03100 México, D.F., México 
a atención médica dirigida (AMD) (managed care) se define como un sistema organizado de atención médica estructurado para obtener la mayor calidad y eficiencia del servicio de salud, ${ }^{1}$ objetivo que se logra mediante la aplicación práctica de conceptos de economía de la salud, economía clínica y farmacoeconomía, sin olvidar nunca la optimización del uso de recursos. Este enfoque es particularmente útil en relación con el empleo de medicamentos, aspecto que cubren de manera específica las organizaciones dedicadas a la administración de beneficios farmacéuticos (ABF) (pharmacy benefit management) con la utilización de formularios, la verificación de elegibilidad con criterios de admisión y surtido predefinidos, el manejo específico de casos y la aplicación de programas clínicos especiales.

Los conceptos de AMD y ABF se forjaron en los Estados Unidos de América (EUA) durante la década de los setenta, y en los tres últimos años se han extendido prácticamente a todo el mundo. ${ }^{2,5}$ En el México de hoy, iniciativas como las mencionadas encuentran terreno fértil por la rápida evolución de los servicios de salud en todos los ámbitos durante los últimos años. Recientemente, Frenk* denominó a este tipo de empresas "organizaciones articuladoras de servicios integrales de salud"(OASIS), descripción que permite enfatizar en forma global el concepto de AMD.

La integración del médico de consultorio a la actividad de las farmacias, parte fundamental de todo sistema de AMD o ABF, permite definir y modificar hábitos prescriptivos como efecto directo de un buen flujo de información médico-farmacia. El médico recibe una retroalimentación efectiva por medio del historial farmacológico del paciente, reglas de uso de medicamento, formularios establecidos, guías de tratamiento y programas clínicos especiales. A los beneficios clínicos innegables de este tipo de programas, se agregan además, en forma implícita, beneficios económicos que resultan de evitar la sobreutilización, el abuso o el uso inapropiado de los medicamentos.

La piedra angular de un programa de AMD o de $\mathrm{ABF}$ es la lista de medicamentos que integran su formulario, entendido éste como el grupo de fármacos disponibles para que los médicos receten a la población en donde opere el programa. Los objetivos planteados en la conformación de cualquier formulario terapéutico determinan la manera en que finalmente se

\footnotetext{
* Frenk J. Una visión estratégica de los servicios de salud, modelo de pluralismo estructurado. Fundación Mexicana para la Salud. Conferencia presentada en el Primer Congreso de Estrategia de Servicios de Salud para el Siglo XXI; 1997 marzo 11; México D.F. Documento no publicado.
}

aplique. En este sentido, puede haber tres tipos diferentes de formularios: a) los destinados únicamente a reembolsos; b) aquellos con un enfoque puramente clínico, y c) una combinación, en la cual se conjugan el objetivo económico-financiero y el clínico. En cualquier caso, en un formulario todos los medicamentos deben clasificarse según criterios preestablecidos, ya sea que se contemple un esquema de seguro médico un programa de atención médica o simplemente que se recomienden ampliamente para una situación clínica específica o para un grupo médico en particular. Un formulario puede ser abierto, es decir, que incluya todos los medicamentos disponibles en el mercado y que sólo establezca recomendaciones para su prescripción, o bien, cerrado, lo que implica aplicar restricciones y exclusiones. ${ }^{1}$ En México existe una experiencia amplia en la utilización de formularios, particularmente con las varias ediciones del Cuadro Básico de Medicamentos del Sector Salud (CBMSS). ${ }^{6} \mathrm{Al}$ evaluar la utilidad de un formulario terapéutico es fundamental valorar asimismo sus objetivos.

Según la International Society for Pharmacoeconomics and Outocomes Research, en un formulario cerrado se controlan de manera estricta los medicamentos disponibles, ${ }^{7}$ y, por lo tanto, el médico prescriptor debe ajustarse a él. En este sentido, el CBMSS es un ejemplo típico de un formulario cerrado, que ha probado ser útil de acuerdo con los objetivos para los que fue creado.

En este trabajo se presenta un formulario abierto diseñado y aplicado en un sistema de ABF en México.

\section{Material y métodos}

En noviembre de 1995 se estructuraron las bases para iniciar un programa de AMD en México para dar servicio inicialmente a una población de 10500 beneficiarios de acuerdo con un esquema de prepago. El tipo de beneficio farmacéutico cubierto por el pagador para esta población incluye 100\% de la prescripción médica $\mathrm{y}$ acceso a una red de servidores privados independientes, (médicos y farmacias) que tienen convenios de prestación de servicios con tabuladores y descuentos preestablecidos. Uno de los aspectos más importantes desde la planeación de todo el programa fue la integración de un comité terapéutico y farmacológico: esto es, un grupo de médicos de diferentes especialidades cuya característica común fue la de ser expertos en su área, con reconocimiento en todo el territorio nacional. A este comité se le encomendó, como tarea inicial, una extensa revisión analítica y crítica de información científica basada en evidencia, -obtenida ésta mediante diferentes fuentes especializadas en medi- 
camentos-, así como elaborar recomendaciones prácticas y útiles para médicos que proporcionan atención a pacientes ambulatorios de la práctica privada en nuestro medio. Desde su formación, este grupo quedó estructurado como un comité científico con un perfil puramente académico, y se constituyó por 11 especialidades seleccionadas según el perfil epidemiológico prevaleciente en México (cardiología, endocrinología, farmacología clínica, gastroenterología, ginecobstetricia, infectología, medicina interna, neumología, oncología, pediatría y reumatología). La principal diferencia de este comité con los que funcionan en EUA* fue que aquí se incluyeron las especialidades de pediatría, ginecobstetricia e infectología, las cuales en nuestro medio evidentemente tienen una gran importancia. Además, se estableció un grupo de apoyo, que se convoca de acuerdo con los tópicos en discusión, constituido por especialidades como dermatología, alergología, oftalmología, hematología, psiquiatría, otorrinolaringología, urología y neurología, lo que permitió cubrir otras áreas importantes y hacer en conjunto recomendaciones clínicas sobre el empleo de más medicamentos.

Para seleccionar a los médicos representantes de cada especialidad se eligió un perfil con base en el $\mathrm{cu}$ rriculum vitae, por lo que en cada caso se realizó un análisis cuidadoso del número de publicaciones nacionales e internacionales, grados y nombramientos académicos, así como de su experiencia personal en medicina privada. Dentro de este marco se logró integrar un grupo interdisciplinario de médicos especialistas que tuvieron en común ser reconocidos a nivel nacional por sus aportaciones académicas y con excepción del farmacólogo clínico todos con intensa práctica médica privada, factores considerados como fundamentales para lograr los objetivos del programa.

Como primera tarea, el comité revisó cada uno de los medicamentos disponibles en el mercado farmacéutico para integrar un formulario que incluyera recomendaciones prácticas para nuestro medio. Se utilizó una modificación de la clasificación de medicamentos propuesta por la American Hospital Formulary Service (AHFS). ${ }^{8}$ El comité discutió cada clase terapéutica de la totalidad de medicamentos disponibles para su venta en México, clasificando al ingrediente activo como: a) esencial, definido como el que ofrece beneficios que lo distinguen del resto de los medicamentos

\footnotetext{
* Guidelines for selection of independent clinical advisory board members, 1995. Documento confidencial de Merck-Medco Managed Care, Inc., EUA.
}

de su misma clase; b) excluido, considerado como aquel en el cual la información recabada es discutible, o en el cual hay evidencia de que su efectividad y seguridad son superadas por otros medicamentos, y c) no esencial-no excluido, definido como uno más en su clase, es decir, con la misma efectividad y seguridad que los demás y sin claras ventajas respecto a los ya existentes. Unicamente se discutieron ingredientes activos; las diferentes formas farmacéuticas y vías de administración sólo se consideraron en casos muy particulares, cuando existían implicaciones clínicas muy importantes.

La primera etapa para la integración del formulario fue analizar el perfil farmacológico y clínico de cada uno de los medicamentos, para lo cual se revisaron estudios clínicos tanto multicéntricos como no multicéntricos, con resultados basados en evidencia pero considerando también la experiencia clínica y el conocimiento farmacológico de cada uno de los miembros del comité. Cuando el comité lo consideró necesario, se realizó una búsqueda bibliográfica extensa y minuciosa en bases de datos especializadas.

La logística de trabajo se definió desde la primera reunión; se nombró un presidente, cuya principal función fue coordinar las sesiones de trabajo mediante la conducción y agilización de las sesiones en cuanto a temática, tiempos y prioridades. Para lograr el objetivo final se estableció que cada decisión se aprobara por el comité en pleno, con un sustento científico necesario y de medicina basada en evidencia (MBE). Se discutió cada medicamento en forma individual hasta consensar su clasificación. Cuando hubo polémica o la discusión se prolongaba demasiado, la decisión final se posponía hasta obtener mayor información. Cada miembro del comité tenía derecho a votar, pero también de abstenerse cuando reconocía no tener experiencia con el medicamento en cuestión.

Durante todo el proceso de evaluación se contó con un paquete de información sobre uso de medicamentos que incluía: a) el análisis de más de 22000 recetas realizadas durante 1995 por médicos que atienden pacientes de la industria bancaria; $b$ ) las bases de datos del Diccionario de Especialidades FarmacéuticasPLM ${ }^{\circledR}$, International Marketing Services (IMS) ${ }^{\circledR}$, Vademecum Farmacéutico ${ }^{\circledR}$, y de importantes distribuidores farmacéuticos en México; c) información de nuevos medicamentos obtenida en una búsqueda electrónica en diferentes fuentes como Micromedix ${ }^{\circledR}$, Drugdex ${ }^{\circledR}$, Martindale ${ }^{\circledR}$ y Physicians Desk Reference (PDR)®; d) información farmacológica y farmacéutica local e internacional; e) el formulario abierto de un programa de AMD vigente en EUA; f) la última versión del CBMSS, ${ }^{6}$ y g) una base de datos con la infor- 
mación recabada a partir de las fuentes anteriores, que sirvió de plataforma para la elaboración del formulario final. Cada miembro del comité recibía -con más de tres semanas de anticipación a las reuniones de trabajo- una carpeta con todo el material bibliográfico relacionado con los temas que se tocarían en las juntas. El comité discutió únicamente ingredientes activos disponibles para su venta en el mercado farmacéutico de México, por lo que, antes de someterlo a discusión en las sesiones de trabajo, se verificó la existencia de cada medicamento.

Aunque no fue posible contar con el Registro de Medicamentos de la Secretaría de Salud, con las bases de datos de los principales distribuidores de medicamentos en nuestro país se facilitó enfocar la evaluación únicamente en los productos efectivamente disponibles en nuestro país.

Debido a que el propósito fundamental de la clasificación de los medicamentos fue la conformación de un formulario universal que sirviera de base para la elaboración de planes para los diferentes esquemas de atención médica con criterios puramente clínicos, en todo momento se evitó de manera intencional mencionar marcas, precios o laboratorios de manufactura. El formulario se aplica en el área médica de la institución que paga por el beneficio farmacéutico, a la cual se le presentan las recomendaciones basadas en la revisión del comité y la que define el plan o listado de medicamentos que finalmente integra su formulario.

Los aspectos más trascendentes de cada reunión eran capturados de manera detallada en una computadora personal por un médico que no pertenecía al comité y que no participaba directamente en la discusión. Esta información se analizaba posteriormente y se resumía para elaborar un documento con las conclusiones de cada junta de trabajo. Ante cualquier duda, siempre se obtuvo una retroalimentación de manera directa con los miembros del comité.

Para el grupo de medicamentos clasificados como excluidos, tuvo lugar una segunda fase antes de su eliminación definitiva: se efectuó una nueva y cuidadosa búsqueda bibliográfica específica y se levantó una encuesta por escrito a una muestra representativa de 80 médicos que prescribían recetas a pacientes de la industria bancaria, para conocer su opinión en relación con la recomendación de exclusión que había hecho el comité. Esta información se utilizó en una segunda sesión de trabajo, en la que se evaluó de nuevo con sumo cuidado el posible impacto de la eliminación definitiva del formulario de este grupo de ingredientes activos, y con la seguridad de que en cada caso existiera evidencia que respaldara la exclusión.

\section{Resultados}

El comité tuvo su primera reunión de trabajo en junio de 1996, y hasta agosto de 1997 se logró clasificar todos los ingredientes activos disponibles en nuestro país. Para llegar a este punto se necesitó una gran cantidad de juntas informales con el fin de consultar con los miembros del comité primario y del grupo de apoyo, así como tres reuniones formales del comité en pleno de dos días de trabajo cada una.

Se evaluó el universo de medicamentos actualmente comercializados en México y disponibles en las farmacias para su venta al público. Esta base contiene un total de 6420 productos farmacéuticos en presentaciones finales, que corresponden a 3810 marcas comerciales y 1211 ingredientes activos individuales o combinados, $100 \%$ de los cuales están disponibles en nuestro país.

Cada uno de los medicamentos se integró para su manejo práctico, en uno de 15 grandes grupos de acuerdo con la especialidad médica a la que pertenecían. Algunos medicamentos se insertaron en más de una especialidad, ya sea por tener una forma farmacéutica diferente o simplemente por razones prácticas para el médico usuario. Se encontró que más de la mitad (50.5\%) de todos los medicamentos disponibles en el mercado farmacéutico mexicano se encuentran concentrados en cuatro especialidades: infectología (14\%), neurología y psiquiatría (14.1\%, aquí están la mayoría de los analgésicos), dermatología (12.1\%) y gastroenterología $(10.3 \%)$.

Del universo de medicamentos evaluados $(\mathrm{n}=$ $1211)$, el comité consideró como medicamentos esenciales, 429 (35.4\%); como excluidos, $105(8.7 \%)$ y como no esenciales-no excluidos, 677 (55.9\%). La distribución de cada tipo de medicamentos por especialidad se observa en el cuadro I. La lista final de medicamentos esenciales -grupo que podría considerarse como el mínimo necesario del programa de AMD- se muestra en el anexo.

Cuando un ingrediente activo era el único representante en México de una clase terapéutica, se clasificó tomando en cuenta la importancia de que dicha clase estuviera representada en el formulario final.

En los casos en que existen dos o más ingredientes activos en una misma clase terapéutica, la clasificación de cada uno de estos medicamentos incluía aspectos basados en evidencia científica que permitieran distinguir un fármaco del resto de la misma clase (p.e., inhibidores de la enzima convertidora de angiotensina). En algunos casos, si no había una marcada diferencia entre distintos fármacos de una misma cla- 


\section{Cuadro I \\ Números totales de ingredientes activos evaluados. Formulario Abierto MÉxico, 1995-1997}

\begin{tabular}{|c|c|c|c|c|c|c|}
\hline Areas & Total (\%) & Esenciales & Excluidos & $\begin{array}{l}\text { senciales-no } \\
\text { xcluidos }\end{array}$ & $\begin{array}{c}\text { Relación } \\
\text { excluidos/esenciales }\end{array}$ & $\begin{array}{l}\text { Relación no esenciales-no } \\
\text { excluidos/esenciales }\end{array}$ \\
\hline Infectología & $181(14.9)$ & 71 & 13 & 97 & .18 & 1.37 \\
\hline O ncología & $51(4.2)$ & 24 & 0 & 27 & 0 & 1.13 \\
\hline N eurología y psiquiatría & $171(14.1)$ & 68 & 6 & 97 & .09 & 1.43 \\
\hline Cardiología & $109(9)$ & 45 & 4 & 60 & .09 & 1.33 \\
\hline Dermatología & $146(12.1)$ & 29 & 19 & 98 & .66 & 3.38 \\
\hline O torrinolaringología & $15(1.2)$ & 5 & 2 & 8 & .40 & 1.60 \\
\hline Endocrinología & $52(4.3)$ & 31 & 4 & 17 & .13 & 0.55 \\
\hline Gastroenterología & $125(10.3)$ & 39 & 15 & 71 & .38 & 1.82 \\
\hline Inmunología & $21(1.7)$ & 18 & 3 & 0 & .17 & 0 \\
\hline Reumatología & $46(3.8)$ & 16 & 4 & 26 & .25 & 1.63 \\
\hline Ginecobstetricia & $75(6.2)$ & 35 & 2 & 38 & .06 & 1.09 \\
\hline O ftalmología & $57(4.7)$ & 16 & 5 & 36 & .31 & 2.25 \\
\hline N eumología & $95(7.8)$ & 15 & 25 & 55 & 1.67 & 3.67 \\
\hline Vitaminas, hematínicos y electrolitos & $49(4)$ & 15 & 1 & 33 & .07 & 2.20 \\
\hline Misceláneos & $18(1.5)$ & 2 & 0 & 16 & 0 & 8.0 \\
\hline Total & 1211 & $429(35.43 \%)$ & b) $105(8.51 \%)$ & $679(56.8 \%)$ & 0.24 & 1.58 \\
\hline
\end{tabular}

se, considerada relevante, se clasificó a todos como esenciales, pues se decidió que la presencia de por lo menos uno de ellos en un formulario cerrado sería suficiente para asegurar la calidad de la atención médica (p.e., antagonistas $\mathrm{H} 2$ ).

Al comparar la distribución por grupos de los ingredientes activos clasificados como esenciales con la lista original (anterior a la clasificación), se observa que la especialidad de dermatología corresponde a $1.63 \%$ del total, contra $12.1 \%$ que tiene en el mercado farmacéutico actual. Esta reducción explica el hecho de que dermatología no aparezca como una de las especialidades con mayor peso en la lista de esenciales, en la cual 51.98\% se concentra en cuatro grupos: infectología $(16.6 \%)$, neurología y psiquiatría $(15.9 \%)$, cardiología $(10.5 \%)$ y gastroenterología $(9.1 \%)$.

El grupo de ingredientes activos excluidos se puede observar también en el anexo. Como puede apreciarse, 25 de los 105 medicamentos de esta clase (24\%) son productos que contienen cuatro o más ingredientes activos, y su exclusión se basó fundamentalmente en el riesgo que implica el posible sinergismo de varios de estos ingredientes ${ }^{9,10} \mathrm{y}$ la falta de evidencia científica que apoyara su efectividad y seguridad. Este criterio afectó particularmente a los medicamentos que pertenecen a tres áreas específicas: a) neumología y alergia, b) dermatología y c) gastroenterología, en donde se concentraron más de la mitad de las exclusiones $(56.19 \%)$, con $23.8,18.1$ y $14.3 \%$, respectivamente.

El grupo de medicamentos catalogados como no esenciales-no excluidos correspondió a 56.97\% del total del universo, y se concentraron primordialmente en cuatro grupos: $14.4 \%$ en dermatología, $14.3 \%$ en infectología, $14.2 \%$ en neurología (grupo que incluye analgésicos) y $10.5 \%$ en gastroenterología (cuadro I).

La relación entre excluidos/ esenciales es más alta en neumología y alergia (1.67), seguida de dermatología (0.66) y otorrinolaringología (0.40), mientras que la relación entre no esenciales-no excluidos/esenciales se encuentra por arriba de 2 en cinco grupos: misceláneos (8.0, aunque con $\mathrm{n}=18$, el número total más bajo de medicamentos), neurología (3.67), dermatología (3.38), oftalmología (2.25) y vitaminas (2.20).

\section{Discusión}

El formulario universal que se presenta en este trabajo ha sido la base para diseñar el plan de medicamentos que conforman el formulario que se está aplicando desde noviembre de 1996 en un programa de AMD en México. La clasificación en este formulario universal, definido así por contener todos los medicamentos disponibles en el mercado farmacéutico mexicano, per- 
mite hacer recomendaciones basadas en evidencia para incluir o excluir medicamentos (grupo de esenciales o de excluidos, respectivamente). El programa en que se aplica este formulario está diseñado para que, antes de ser surtida, cada receta se compare siempre con una base central de datos farmacológicos que incluye el historial farmacológico del paciente y recomendaciones actualizadas y prácticas sobre uso de medicamentos. El sistema, además de vigilar la aplicación del formulario para evitar el surtido de medicamentos catalogados como excluidos, permite detectar situaciones de alto riesgo para el paciente, como interacciones graves de medicamentos, tomar precauciones para pacientes en edad pediátrica o geriátrica, evitar duplicaciones terapéuticas, etcétera, información que se hace llegar al médico prescriptor por medio de una terminal de computadora en su consultorio o de un mensaje codificado desde la farmacia. De esta forma, el médico recibe información antes de que el paciente obtenga el medicamento, lo cual asegura la selección óptima de un medicamento para cada paciente en una situación particular. Cada médico tiene un manual que incluye el listado completo de medicamentos con su clasificación respectiva, donde se informa además sobre la política de cobertura de medicamentos que aplica la institución patrocinadora del beneficio farmacéutico. El sistema permite detectar al médico que elabora recetas con medicamentos catalogados como excluidos y planear intervenciones que modifiquen su perfil prescriptivo.

Para que todo esto se lleve a cabo de una manera dinámica, se requiere tener en forma permanente un listado completo de los medicamentos disponibles regularmente en las farmacias, integrados en una base de datos electrónica que debe actualizarse periódicamente (en México, debido a la frecuencia en la variación de los precios de medicamentos -25 a 30 cambios diariamente- y en la entrada de nuevos productos -normalmente más de 100 al mes-, se recomienda que la actualización sea diaria). Esta base de datos debe ser altamente confiable y estar siempre a disposición del médico prescriptor.

El formulario propuesto en este trabajo proviene del universo de medicamentos actualmente comercializados en México y disponibles en las farmacias para su venta al público. Se han desarrollado formularios similares en países industrializados y no industrializados; por ejemplo, en EUA existen aproximadamente 5000 productos diferentes (marcas comerciales), los cuales se encuentra incluidos en una publicación periódica con información farmacéutica actualizada. En Argentina existen alrededor de 7000 productos, también integrados en un manual. En España hay alrede- dor de 16000 medicamentos, incluidos en una base de datos no comercial al alcance de cualquier usuario.* En este sentido, México se encuentra en franca desventaja, pues a pesar de que se tiene un número importante de productos farmacéuticos, no existe una fuente de información actualizada, confiable y sin sesgos comerciales que permita al médico en la práctica privada seleccionar un producto farmacéutico con la certeza de que esté disponible y que lo ayude a reducir los frecuentes errores que genera la falta de información al prescribir. Este trabajo puede ser el primer paso hacia ese objetivo.

Los números finales de este proceso de evaluación de medicamentos son interesantes. Aproximadamente uno de cada 10 ingredientes activos disponibles en el mercado farmacéutico en México se clasificó como excluido, mientras que casi cuatro de cada $10(38.8 \%)$ se catalogaron como esenciales. En otras palabras, del total de medicamentos que es posible encontrar en las farmacias de nuestro país sólo alrededor de $40 \%$ se consideraron alternativas que aportan un valor indiscutible en la prescripción médica, mientras que el uso de $10 \%$, aproximadamente, significaría un riesgo potencial para el paciente. En el mismo sentido, más de la mitad de los ingredientes activos disponibles comercialmente en México (55.9\%) se consideraron como uno más (del inglés me-too) en sus respectivas clases, sin un valor agregado que los haga realmente indispensables. Este grupo de medicamentos es, sin duda, el que representa mayor gasto y problemas terapéuticos. La relación entre este grupo y el de esenciales muestra que prácticamente para cada medicamento esencial existe por lo menos un no esencial-no excluido (promedio de 1.58), y que existen por lo menos cuatro grupos en donde hay más de dos de estos potenciales sustitutos por cada ingrediente activo esencial (neurología, dermatología, oftalmología y vitaminas, con 3.7, $3.4,2.3$ y $2.2 \%$, respectivamente) (cuadro I), lo que sin duda contribuye al incremento constante en el gasto de medicamentos.

El principal motivo para excluir un medicamento fue la asociación de varios ingredientes activos en el mismo producto (uno de cada cuatro de los que el comité recomendó excluir eran productos cuya formulación contenía cuatro o más ingredientes activos), combinación que no ofrece ningún beneficio comprobado por MBE. La puesta en marcha de estas recomendaciones podría contribuir a abatir la polifarmacia,

\footnotetext{
* Manual operativo de Merck-Medco Managed Care, Inc., EUA, 1997. Documento confidencial.
} 
factor común pero poco reconocido de sobreutilización de medicamentos en la práctica médica privada.

Un índice tal vez útil para distinguir los grupos terapéuticos que concentran la mayoría de los productos farmacéuticos comerciales cuyo uso implica un mayor riesgo, según el criterio del comité de expertos, lo constituye la relación entre excluidos/esenciales (cuadro I). Por ejemplo, el índice de 1.67, correspondiente al grupo de neumología y alergia, implica que existen en el mercado farmacéutico mexicano más medicamentos potencialmente peligrosos (excluidos) que medicamentos que aportan un valor terapéutico indiscutible (esenciales).

Por otra parte, es importante aclarar que el término esencial, definido por la Organización Mundial de la Salud $(\mathrm{OMS})^{11}$ se refiere al medicamento que satisface las necesidades de salud de la mayoría de la población, por lo que debe estar siempre disponible en cantidades y presentaciones farmacéuticas adecuadas. Según este criterio, la selección de los medicamentos esenciales depende de múltiples factores, que de ninguna manera se consideraron en el presente trabajo (véase la sección "Material y métodos"), por lo que el grupo de medicamentos catalogados por la OMS como esenciales no puede compararse con el grupo de esenciales resultante de esta evaluación.

De la misma manera, como los objetivos y la utilización del CBMSS son diferentes a los planteados aquí, su listado de medicamentos y la manera como se clasifican no son comparables con el grupo de esenciales propuesto. Sin embargo, al analizar la última versión del CBMSS, ${ }^{6}$ que contiene un total de 509 ingredientes activos -de los cuales $163(32 \%)$ han sido clasificados como de primer nivel-, se observa que $100 \%$ de ellos también han sido clasificados como esenciales en el formulario universal que aquí se propone.

En conclusión, este formulario abierto para pacientes ambulatorios, estructurado por un comité científico del más alto nivel, constituye una herramienta útil para que el médico pueda tomar una decisión terapéutica más apropiada para su paciente, segura, efectiva, sin restricciones y dentro del marco del más alto control de calidad en los medicamentos, lo que permitirá ofrecer una atención médica integral completa. Las características de este formulario, debido a los objetivos de su conformación, difieren con claridad de otros utilizados en medicina institucional. ${ }^{11}$ Una segunda fase de este programa se enfocará en la revisión del formulario tomando en cuenta aspectos de farmacoeconomía como complemento de la evidencia clínica, la cual fue la base de esta primera evaluación.

\section{Agradecimientos}

El Comité Terapéutico y Farmacológico de México se constituyó con el apoyo de la compañía Medco de México Managed Care, S. de R. L. de C.V. Las siguientes personas participaron directamente en todo el proceso descrito en este trabajo: doctores Ingris Peláez Ballestas, Artemisa Aguilar Chiu y Gilberto Castañeda Hernández. Grupo de apoyo: doctores José Clemente Díaz Maqueo (hematología), Salvador González Gutiérrez (psiquiatría), José Huerta López (inmunología y alergia), Gladys León Dorantes (dermatología), Mario Antonio Mandujano Valdez (otorrinolaringología), Francisco Martínez Castro (oftalmología), Francisco Rubio Donnadieu (neurología), Aarón Torres García (urología).

\section{Referencias}

1. Glossary. Pharmacoeconomics 1997;11(6):610-613.

2. Kassirer,W. Is managed care here to stay? N Engl J Med 1997;336(14): 1013-1017.

3. Ginzberg T, 0 stow P. Managed care. A look back and a look ahead. N Engl J Med 1997;336(14):1018-1021.

4. Epstein RS, McG lynn MG. D isease management.W hat is it? D is Manage Health O utcomes 1997;1(1):3-10.

5. Mossialos E, Kanavos P, A bel-Smith B.W ill managed care work in Europe? Pharmacoeconomics 1977;11(4):297-305.

6. Cuadro Básico de Medicamentos del Sector Salud. Diario 0 ficial de la Federación 1996 noviembre 15.

7. Pashos CL, Klein EG, W onke LA, eds. ISPO R LEXIC O N . N ueva Jersey: International Society for Pharmacoeconomics and 0 utcomes Research, 1998:125.

8. American Hospital Formulary Service. Drug Information. Bethesda: A merican Society of Health-System Pharmacists, 1995.

9. Colley CA, Lucas LM. Polypharmacy:The cure becomes the disease. J Gen Intern Med 1993;8(5):278-283.

10. Carlson JE. Perils of polypharmacy: 10 steps to prudent prescribing. Geriatrics 1996;51(7):26-30.

11.The use of essential drugs. Sixth report of theW HO Expert C ommittee. (W HO Technical Report Series, núm. 850). Ginebra: World Health 0 rganization (W HO Techical Report Series, núm. 850), 1995. 


\section{Anexo \\ INGREDIENTES ACTIVOS ESENCIALES (MARCADOS CON NEGRITAS) Y NO ESENCIALES-NO EXCLUIDOS*}

1. Infectología

aciclovir

albendazol

amikacina

amoxicilina

amoxicilina/ambroxol

amoxicilina/bromhexina

amoxicilina/clavulanato

ampicilina

ampicilina/sulbactam

anfotericina B

azitromicina

aztreonam

bacampicilina

brodimoprina

carbenicilina indanyl

cefaclor

cefadroxil

cefalexina

cefalotina

cefamet pivoxil

cefazolina

cefepime/arginina

cefixima

cefodizima

cefonicid

cefonicid/ lidocaína

cefoperazona

cefotaxima

cefpirome

cefpodoxima

cefprozil

cefradina

ceftazidima

ceftibutén

ceftizoxima

ceftriaxona

ceftriaxona/lidocaína

cefuroxima (acetil)

cinoxacino

ciprofloxacino

claritromicina

clindamicina

cloramfenicol

cloroquina

clorosalicilamida

clortetraciclina

clotrimazol

dapsona

decaliniob

dehidroemetina

demeclociclina

dicloxacilina

didanosina (ddl)

diyodohidroxiquinoleína

diyodohidroxiquinoleína/ metronidazol

diyodohidroxiquinoleína/dimeticona

doxiciclina

enoxacino

\author{
eritromicina base \\ eritromicina, etilsuccinato \\ eritromicina, etilsuccinato/sulfisoxazo \\ espectinomicina \\ espiramicina \\ espiramicina/metronidazol \\ esporfloxacino \\ estreptomicina \\ etambutol \\ etofamida \\ fenazopiridina \\ fluconazol \\ fosfomicina \\ furazolidona \\ furazolidona/colistina \\ ganciclovir \\ gentamicina \\ griseofulvina \\ hemezol \\ imipenem/cilastatina \\ indinavir, sulfato de \\ isepamicina \\ isoconazol \\ isoniazida \\ isoniazida/etambutol \\ isoniazida/rifampicina \\ itraconazol \\ itraconazol/secnidazol \\ kaínico,ácido/ piperacina, adipato \\ de/santonina \\ kanamicina \\ ketoconazol \\ lamivudina \\ lomefloxacino \\ loracarbef \\ mebendazol \\ meropenem \\ metenamina/atropina \\ metronidazol /sodio, cloruro de \\ metronidazol/escopolamina \\ miconazol \\ minociclina \\ miocamicina \\ nalidíxico, ácido \\ nalidíxico, ácido /fenazopiridina \\ neomicina \\ netilmicina \\ nifuratel \\ nifuratel/nistatina \\ nistatina \\ nitazoxanida \\ nitrofurantoína macrocristales \\ norfloxacina \\ ofloxacino \\ omoconazol \\ ornidazol \\ oxitetraciclina \\ paraminosalicílico, ácido (PAS) \\ pefloxacino
}

penicilina benzatínica

penicilina benzatínica G /penicilina

G potásica/penicilina G sódica

penicilina benzatínica $\mathbf{G}$ /penicilina

G sódica

penicilina G potásica/penicilina G

procaínica/penicilina G sódica

penicilina G sódica

penicilina G sódica/procaínica

penicilinaVK

pentamidina isotionato

pipemídico, ácido

piperacilina

piperacilina/tazo bactam

piperacina

pirantel

pirazinamida

pirimetamina

praziquantel

propicilina

proxetil/cefpodoxima

quinfamida

ribavirina

rifampicina

rifampicina/isoniacida/pirazinamida

ritonavir

rosoxacino

roxitromicina

secnidazol

stavudina

sulbenicilina/mepivacaína

sulfaleno/trimetoprima

sulfametoxazol/trimetoprim

sulfasalazina

sulfisoxazol/eritromicina, etilsuccinato

sultamicillina tosilato

teicoplanina

terbinafina

tetraciclina

ticarcilina/clavulanato

tinidazol

tioconazol/tinidazol

tobramicina

tromantadina

valaciclovir, clorhidrato de

vancomicina

zalcitabina (ddC)

zidovudina (AZT)

2. Oncología

actinomicina

asparagina

azatioprina

bicalutamida

bleomicina

busulfano

carbacina

carboplatino

carmustina

\section{(Continúa)}

\footnotetext{
* Algunos ingredientes activos aparecen en más de una sección
} 
Anexo

(Continuación)

\section{ciclofosfamida \\ ciclosporina}

ciproterona, acetato de

cisplatino

citarabina

clorambucilo

daunorubicina

docetaxel

doxorrubicina

epirrubicina

etopósido

filgastrim (G-CSF)

fluorouracilo(5)

flutamida

fosfetrol tetrasódico

gemcitabina

glicofosfopeptical

goserelina

goserelina, acetato de

granisetrón

hidroxiurea (hidroxicarbamil)

ifosfamida/mesna

irinotecán

leuprolide

levamiso

lomustina

megestrol

melfalán

mercaptopurina

metotrexato

mitomicina

mitoxantrona

molgramostim

munomonab-CD 3

octreótido

ondansetrón

paclitaxel

procarbacina

tamoxifén

timostimulina (TP-1)

toremifeno

triptorelina, acetato de

tropisetrón

vinblastina

vincristina

vinorelbina

3. Neurología y psiquiatría acetaminofén/codeína acetilsalicílico, ácido

acetilsalicílico, ácido/cafeína

acetilsalicílico, ácido/aluminio/magnesio

acetilsalicílico, ácido/calcio,carbonato de

/citrico, ácido

acetilsalicílico, ácido/paracetamol/cafeina

alprazolam

amitriptilina

amoxapina

antiinflamatorios no esteroideos

barbital

betahistina

biperideno

bornaprina

bromazepam bromocriptina, mesilato

brotizolam

buprenorfina

buspirona

butiluinal

butorfanol

butriptilina

cafeína/efedrina/paracetamol

carbamacepina

carbidopa-levodopa

cinarizina

citalopram

clobazam

clomipramina

clonacepam

clonixina/escopolamina

clonixina/pargeverina

clonixinato de lisina

cloracepato dipotásico

clorodiacepóxido

clorodiacepóxido/clidinio

clorpromazina

clorzoxazona/paracetatol

clozapina

co-dergocrina, mesilato de

demeclozina/piridoxinio

desipramida

diazepam

difenhidramina

difenidol

diflunisal

dihidroergocristina

dihidroergotamina/cafeína/propifenazona

dihidroergotamina/paracetamol cafeína

dimenhidrinato

dimetotiacida

divalproato sódico

doxepín

droperidol

ergotamina, tartrato de/acetilsalicílico, ácido/cafeína

ergotamina/cafeína

estazolam

etosuximida

fenitoína

fenobarbital

fenobarbital/belladona

fenobarbital/fenitoína

fenobarbital/fenitoína/aminohidroxibutírico, ácido

fentermina

flufenacina/nortriptilina

flumazenil

flunaricina

flunitracepam

fluoxetina

fluvoxamina

gabapentina

granisetrón

haloperidol

hidroxicina

hidroxicina/buclicina/ácido nicotínico

hipericum

imipramina ketoprofeno/paracetamol

ketorolaco

lamotrigina

levodopa-benserasida

levomepromazina

litio, carbonato de

loflacepato de etilo

lomifilina/mesilato de dihidroergocristina

lorazepam

lormetazepam

magnesio, valproato de

maprotilina

meclizina/piridoxina

meperidina (petidina)

metamizol

metamizol/cianocobalamina/piridoxina/tiamina

metamizol/cianocobalamina/piridoxina/ tiamina/lidocaína

metamizol/clorfenamina/lidocaína/clorhexidina

metamizol/escopolamina

metamizol/papaverina

metilfenidato

metocarbamol

metocarbamol/ acetilsalicílico, ácido

metocarbamol/paracetamol

metotriperacina

mianserina

midazolam

moclobemida

morfina, sulfato

nalbufina, clorhidrato de

naproxén/lidocaína

neostigmina

ondansetrón

orfenadrina

orfenadrina/paracetamol

oxcarbazepina

paracetamol

paroxetina

penfluridol

perfenacina/amitriptilina, clorhidrato de

perfenazina

pergolida, mesilato

pipotiazina

piridostigmina

pizotifeno

primidona

propifenazona/paracetamol/cafeína

propoxifeno, clorhidrato de

propoxifeno/acetilsalicílico, ácido /cafeína propoxifeno/paracetamol

risperidona

selegilina

sertralina

sulpirida

sumatriptán, succinato

tetrazepam

tiaheptina

tiocolchicósido/cobamamida

tioridazina

tizanidina, clorhidrato

tolperisona/paracetamol

toxina botulínica tipo A

tramadol

(Continúa) 
Anexo

(Continuación)

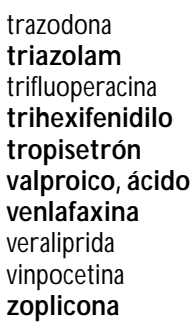

4. Cardiología acenocumarol acetilsalicílico, ácido acipimox amilorida/furosemida aminocaproico, ácido amiodarona

amlodipino

atenolol

atenolol/clortalidona

benazepril

benciclano, fumarato ácido

bezafibrato

bufenina

bumetanida

captopril

captopril/hidroclorotiazida

carvedilol

cilazapril

ciprofibrato

clonidina

clortalidona

clortalidona/oxprenolol, clorhidrato de clortalidona/atenolol

colestipol

colestiramina

cumarina/troxerrutina

di-isoprilamonio, dicloroacetato de

digoxina

diltiazem

diosmina/hesperidina

dipiridamol

disopiramida

dobesilato de calcio

enalapril

enalapril/hidroclorotiazida

enoxaparina

esmolol

estreptoquinasa

etilefrina, clorhidrato de

felodipino

fenobibrato

fitomenadiona

fitomenadiona/ carbazocroma

flecainida

fluvastatina

fosinopril

furosemida

gallopamilo

gemfibrosil

glicerilo, trinitrato de

heparina

hidroclorotiacida hidroclorotiacida/amilorida/timolol

hidroclorotiacida/amilorida

isosorbida, dinitrato

isosorbida, dinitrato de (spray)

isosorbida, dinitrato de (sublingual)

isosorbida, mononitrato

isoxsuprina

isradipino

ketanserina

lacidipino

lisinopril

lisinopril/hidroclorotiazida

losartán

losartán/hidroclorotiacida

lovastatina

mepindolol, sulfato de

metoprolol

metoprolol/hidroclorotiacida

mexiletina

nadolol

nadolol/bendroflumetiacida

naftidrofurilo

nicardipino

nicergolina

nicotínico, ácido

nifedipino

nimodipina

nisoldipino

nitrendipino

nitroglicerina

nordroparina

norfenefrina, clorhidrato

papaverina

pentoxifilina

perindopril

pindolol

pindolol/clopamide

potasio, bicarbonato de/potasio, cloruro de/cítrico, ácido

pravastatina

prazosina

propafenona

propranolol

quinapril

quinidina, poligalacturonato

quinidina/feno barbital/valerian

ramipril

simvastatina

sotalol

terazosina

ticlopidina

timolol

torasemida

trandolapril

triamtereno/hidroclorotiacida

trifusal

uroquinasa

verapamilo

warfarina

5. Dermatología

ác.mineral/alcohol cetílico/sorbitán/alcohol esteárico/ sorbitol

aceite sulfonado/aceite mineral/antioxidantes acexámico, ácido

acexámico, ácido/neomicina

acicetrino

aciclovir

acrilato, copolímero de/glicerina

alcanfor/mentol

alkilamina betaína

almendras dulces, aceite de

alquitrán de hulla

alquitrán de hulla /alantoína

alquitrán de hulla/ hidantoína

alquitrán de hulla/mentol

alquitrán de: pino, junípero y hulla/alcohol oleico/aráquida

aluminio, clorhidróxido

aluminio, óxido de/triclosán

aluminio,hidróxido de/calamina/benzalconio/ vitaminas $\mathrm{A}$ y $\mathrm{E}$

amcinonida

amonio, lactato

amorolfina

antralina

aráquida, aceite $2 \%$ /mexiloxiestearato $1.5 \%$ lecitina

asiaticósido

azelaico, ácido

azufre/ácido salicílico

benzoílo, peróxido

betametasona, valerianato

betametasona/yodoclorhidroxiquinoleína

bifonazol

bifonazol/urea

bucotoconazol

calamina

calcio, acetato de/aluminio, sulfato de

calcio, hidróxido de /aceite de almendras

calciprotiol

capsaisina

carbamida (urea)

ciclopirox

ciproterona, acetato de

clindamicina

clioquinol

clioquinol/alantoína/alquitrán de hulla

clioquinol/alquitrán de hulla/alantoína/triclosán

clobetasol

clotrimazol

clostebol

cobre, oleato

cobre, sulfato de /sulfato de zinc/alcanfor copolímeros hidrolizados de polisacáridos poliacrilonitrilos

crotamitón

decatrina

dermolimpiador libre de lípidos

dextranómero

diflucortolona valerianato de

diflucortolona/clorquinaldol

dihidroacetona/alcohol /acetona y agua destilada

dimeticona/petrolato

dipropionato de alclometasona

ditranol

econazol

\section{(Continúa)}




\section{Anexo \\ (Continuación)}

eritromicina tópica

fibrinolisina/desoxirribonucleasa/cloranfenicol

fluocinolona, acetónido de

fluocinolona/clioquinol

fluocinónido

fluocinónido/procinonida/ciprocinonida

fluocortolona caproato/fluocortolona pivalato

fluorouracilo

fusídico, ácido

gentamicina

glicerina/mineral,aceite/iso propil palmitato/ agua

glicerina/petrolato/alcohol/xantina/dimeticona/ colesterol/...

glicólico, a./agua/triglicérido caprílico/ propilenglicol/amonio/estearato

halcinonida

hidrocoloide, apósito

hidrocortisona, butirato

hidrocortisona/clio quino

hidroquinona

hígado de bacalao, aceite de/benzalconio/

ox.de zinc/alantoína

higroplex (complejo humectante)

isoconazol

isotretinoína

jabón de avena

ketanserina

ketoconazol

lactato de amonio $12 \%$

láctico, ácido . pH5

láctico, ácido/pirrolidona, carboxilato de

lanolina/parafina líquida

láurico, ácido/ácido esteárico/oleico, ácido/ hidróx de sodio

lauril-éter sulfato de sodio

lecitina vegetal/emolientes y humectantes

lindano

lindano/benzilo, benzoato de

lindano/lidocaína

manzanilla, flores de

metoxalén

metoxicinamato/titanio/vitamina E/glicerina

metoxinamato/homosalato/oxibenzona

miconazol

mometasona, furoato

mupirocina

nistatina

nitrofurazona

omoconazol

oxiconazol

PABA Factor 4

pantoteico, ácido/urea/ricino, aceite de/isopropil palmitato

parsol MCX/benzofenona-3

parsol MCX/benzofenona/titanio

parsol MCX/octocrileno/titanio/parsol1789

parsol MCX/parsol 1789/octocrileno

permetrina

podofilina

polividona-iodo

prótidos, solución coloidal de

resorcina/calamina resorcinol/azufre/triclosán

retinaldehido

ribavirina

rifamicina

salicílico, ácido/ hidantoína

salićlico, ácido/láctico, ácido

soya, harina/polivinilpirrolidona

sulconazol, nitrato de

sulfadiacina de plata

terbinafina

timol/cloruro de benzalconio

tolciclato

tretinoína (ácido retinoico)

tromantadina

urea/metilparabeno/propilenglicol/ac. mineral/ octilpalmitato/.

vaselina

vitamina A y D/hígado de bacalao/zinc ox./ benzalconio

vitamina A/zinc, óxido de/alantoína/ácido bórico/talco

vitaminaA/zinc,óxido de/alantoína/benzalconio, cloruro de

zinc, óxido de

zinc, piritionato

6. Otorrinolaringología

beclometasona

benzocaína tópica

cromolín nasal

dequalinium clorado

dexametasona

dexametasona/neomicina

dexametasona/neomicina/ fenilefrina

hidrocortisona/cloranfenicol/benzocaína

iodopovidona

lidocaína gel

nifuroxima/hidrocortisona

oximetazolina

triamcinolona

7. Endocrinología

acarbosa

alendronato

anfepramona

bromocriptina

calcitonina de salmón

calcitriol

clobenzorex

clomifeno

clorpropamida

danazol

desmopresina, acetato (h. antidiurética)

dexametasona

dextrofenfluraminafentermina

finasteride

fluoximesterona

glibenclamida (gliburida)

gliclazida

glipicida

glucagon

gonadorelina

insulina de res o cerdo insulinahumana(todaslascombinaciones)

levotiroxina

levotiroxina sódica/liotironina sódica

liotironina

mazindolmesterolona

metformina

metimazol

nafarelina

nandrolona, decanoato

octreotida, acetato

pergolida

prednisona

quinagolida, clorhidrato de

somatropina

somatropina (h. de crecimiento)

testosterona

tolbutamida

triyodotironina/tiroxina

urofolitropina

8. Gastroenterología

5-adenosil-metionina

alginato/aluminio/magnesio aloglutamol

aluminio/magnesio

aluminio/magnesio/dimeticona/calcio, carbonato de

amilasa/lipasa/proteasa

benzoato de sodio

betahistina

bisacodilo

bismuto subsalicilato de

bismuto/pepsina

buclizina

butilhioscina

butilhioscina/lisina, clonixato de

butilhioscina/metamizol

calcio y sodio, carbonatos de/hidróxidos de magnesio y aluminio

cimetidina

cisaprida

citrato de sodio/laurilsulfoacetato

clorodiacepóxido/clidinio

cobamamida

dextropantotenato de calcio/dihidroxiantraquinona

dicicloverina

difenidol

difenoxilato/ atropina

dimenhidrinato

dimeticona

dimeticona/aluminio/magnesio

dimeticona/bromelín/pancreatina/extracto de bilis de buey

dimeticona/diyodohidroxiquinoleína

dimeticona/pancreatina

dimeticona/pantotenato de calcio

docusato sódico/fenolftaleína

docusato sódico/sorbitol

domperidona

drofenina/propifenazona

etomidolina

famotidina

(Continúa) 
Anexo

(Continuación)

fenitopentol/benoctidín

fenitopentol/benoctidín/tiamina/nicotinamida

fenoverina

floroglucinol/trimetilfloroglucinol

fluocinolona/óxido galato de bismuto/lidocaína

flucortolona/ cincocaína

glicerina

granisetrón

hidrocortisona/lidocaína/zinc/aluminio

hidrotalcita

himecromona

isopropamida, yoduro de/trifluoperazina, clorhidrato de

lactulosa

lansoprazol

levadura

lidamidina

lisina, clonixinato de

loperamida

magaldrato/dimeticona

magnesio, hidróxido de

magnesio, hidróxido de/aluminio, hidróxido de/oxetacaína

meclizina, clorhidrato de/piridoxina, clorhidrato de

mesalazina (ácido 5 aminosalicílico)

metamizol/clorhidrato de papaverina

metamizol/ pitofenona fenpiverino

metoclopramida

metoclopramida/dimeticona/papaína

metoclopramida/dimeticona

metroclopramida/simeticona/pancreatina/

bromelina

mineral, aceite/fenolftaleína

misoprostol

nifurzida

nifurzida/pectina

nizatidina

omeprazol

ondansetrón

pancreatina

pancreatina/extracto de bilis de buey/celulasa/ dimeticona

pancreatina/hemicelulasa/dimeticona/ extracto de bilis de buey

pantoprazol

paracetamol/hioscina, butilbromido de

pargaverina

pargaverina/metamizol

pargaverina/lisina, clonixato de

pinaverio

pirenzepina, clorhidrato de

plantago ovata/ispagula husk

plantago ovata/senósidos A y B

policarbófilo cálcico

policresuleno/cincocaína

prednisolona/cincocaína

psyllium plantago/dextrosa

psyllium/salvado

quenodesoxicólico, ácido

ranitidina

sen

sen, polvo/tamarindo, caña /ciruelas y manzanas, pulpa silimarina

sodio, picosulfato de

sucralfato

sulfasalazina

tietilperazina, maleato de

tribenósido

tribenósido/lidocaína

trimebutina

tropisetrón

ursodesoxicólico, ácido

zaragatona india, semilla de

9. Inmunología

alfa $2 a$ interferón

alfa $2 b$ interferón

alfa n3 interferón

anti-difteria/tos ferina y tétanos, vacuna

anti-difteria/tos ferina/tétanos y $\mathrm{H}$.

influenzae tipo B

anti-H. influenzae tipo B + DPT

anti-H. influenzae tipo B. Meningitis

anti-haemophilus B (conjugada)

anti-hepatitis A

anti-hepatitis B

anti-hepatitis B (recombinante)

anti-influenza (gripe)

anti-neumocócica polivalente

anti-parotiditis

anti-pertussiscon DPT (vacuna acelular)

anti-poliomielítica

anti-rubéola+neomicina

anti-sarampión

anti-sarampión/parotiditis y rubéola

beta $\mathbf{l b}$, interferón

eritropoyetina

filgastrim (G-CSF)

gammaglobulina humana

molgramostim (GM-CSF)

tétanos, toxoide adsorbido

timomodulina

10. Reumatología

acemetacina

acetil salicílico, ácido

alopurinol

auranofín

azatioprina

benzobromarona

betametasona

ciclofosfamida

ciclosporina A

cloprednol

colchicina

deflazacort

diclofenaco

diclofenaco potásico

diflunisal

etodolac

etofenamato

fenbufén

fenoprofeno cálcico

flurbiprofén

glucametacina

hidrocortisona ibuprofeno

indometacina

ketoprofeno

mefenámico, ácido

meloxicam

mercaptopurina (6)

metilprednisolona

metotrexato

nabumetona

naproxén

naproxén/paracetamol

parametasona

penicilamina-D

piroxicam

prednisona

probenecid

sulfasalazina

sulindac

tenoxicam

tiaprofénico, ácido

tolfenámico, ácido

tolmetín

triamcinolona

trietanolamina, salicilato de

11. Ginecología y obstetricia

alilestrenol

bromocriptina

butaconazol

ciclofenil

ciproterona, acetato de

clindamicina

clomifeno

clormadinona

clotrimazol

danazol

desogestrol-etinil estradiol

dihidroxiprogesterona/estradiol

dinoprostona

estradiol

estradiol, 17-beta

estradiol, benzoato de

estradiol, benzoato de/progesterona

estradiol, benzoato de/hidroxiprogesterona, caproato

estradiol, cipionato de/medroxiprogesterona

estradiol, enantato de

estradiol, enantato de/algestona, acetofenido de

estradiol, valerianato de

estradiol, valerianato de/ciproterona, acetato de

estradiol, valerianato de/hidroxiprogesterona, caproato

estradiol, valerianato de/medroxiprogesterona, acetato de

estradiol, valerianato de/noretisterona, enantato de

estradiol, valerianato de/noretindrona, enantato de

estradiol, valerianato de/norgestrel

estradiol, valerianato/hidroxiprogesterona

estradiol, valerianato/ciproterona

(Continúa) 


\section{Anexo \\ (Continuación)}

estradiol/noretisterona, acetato

estradiol/noretisterona, acetato de

estradiol/norgestrel

estriol, succionato

estrógenos conjugados

estropipato

etinilestradiol/ciproterona, acetato de

fenoterol

formoterol

gestodeno/etinilestradiol

gestrinona

gonadotropina coriónica

hidralazina*

hidroxiprogesterona

indometacina

inmunoglobulina anti Rh

iodopovidona

isoconazol

isoxsuprina

leuprolida

levonorgestrel

levonorgestrel/etinilestradiol

linestrenol

lisurida, hidrogenmaleato

medrogestona/estrógenos conjugados

medroxiprogesterona, acetato de

medroxiprogesterona/estradiol

menotropinas

mestranol/clormadinona, acetato de

metandriol, dipropionato de/estradiol, benzoato de/progest.

metildopa*

metilergometrina

metronidazol/nistatina

miconazol

nafarelina

nonoxinol-9

nonoxinol-9/cloruro de metilbencetonio

nonoxinol/éster poliácido sulfúrico de

noretisterona/mestranol

noretindrona

noretindrona-etinilestradiol

noretindrona-mestranol

norgestimato-etinilestradiol

norgestrol-etinilestradiol

orciprenalina

piperidolato, clorhidrato de

policresuleno

prasterona/estradiol

progesterona micronizada

terconazol

testosterona, cipionato/estradiol,cipionato de

testosterona, cipionato/testosterona,

propionato/prasterone

tibolona

triticum repens/fenoxietanol

undecilénico, ácido /zinc, undecilenato

urofolitropina

veralipride

* Se recomienda su uso en HTAS del embarazo

\section{Oftalmología}

acetazolamida

aciclovir

alcohol, polivinil

alcohol, polivinil/polividona

antazolina/nafazolina

atropina, sulfato de

betaxolol

ciclopentolato

ciprofloxacina

cromoglicato de sodio

dexametasona

dexametasona/tobramicina

dexametasona/cloranfenicol

dexametasona/fenilefrina

dexametasona/neomicina

diclofenaco

dipivefrina

dorzolamida, clorhidrato

eritromicina

fenilefrina, clorhidrato de/sodio, borato/ bórico, ácido

fenilefrina, clorhidrato de /sodio, borato/ dexametasona, fosfato

fenilefrina/metilcelulosa

feniramina, maleato de/nafazolina

fluorometalona

fluorometalona/tetrahidrolizina

flurbiprofeno

gentamicina

homatropina

ketorolaco

lanolina/ aceite mineral/petrolatum, blanco

levobunolol

levocabastina

metilcelulosa (lágrimas artificiales)

metipranolol, buticida

nafazolina

nafazolina/antazolina

norfloxacina

ofloxacina

oximetazolina/alcohol polivinílico

oxitetraciclina, clorhidrato de/polimixina B, sulfato de

pilocarpina $\mathrm{Hcl}$

potasio, cloruro de/sodio, cloruro de

prednisolona

prednisolona (fosfato de sodio)

prednisolona/cloranfenicol

prednisolona/cloranfenicol/benzalconio

proparacaína

sodio, cloruro de/sodio, borato de /halethazole

sodio, hialuronato de

sulfacetamida

sulfacetamida/prednisolona/fenilefrina/alcohol polivinílico

sulfacetamida/prednisolona

sulfacetamida/prednisolona/neomicina

tetracaína

tetraciclina

tetrahidrozolina, clorhidrato de/polietileno glicol

timolol

tobramicina tropicamida

vidarabina

zinc, sulfato de/fenilefrina

zinc, sulfato de/nitrato de nafazolina

zinc/fenilefrina

13. Neumología y alergia

acetilcisteína

aminofilina

astemizol

astemizol/pseudoefedrina

azatadina

azelastina

beclometasona

benzonatato

bromofeniramina

bromhexina/oxeladina

bromofeniramina, maleato de

bromofeniramina/fenilpropanolamina

bromhexina/orciprenalina

bromhexina/oxeladina

budesonida

carbocisteína

cetirizina

clemastina

clenbuterol

clorfenamina, maleato de/fenilpropanolamina

clorfenamina/paracetamol

clorfenamina/salicilamida

clorfeniramina

clorfeniramina/acetaminofén/cafeína/fenilefrina

clorfeniramina maleato

clortenoxazina/clorfenamina

cromoglicato

dexametasona

dexclorfeniramina

dextrometorfano

dextrometorfano/guaifenesina

dextrometorfano/guayacol

difenhidramina

difenhidramina/amonio y sodio

dropropizina

efedrina/bálsamo de tolú

erdosteína

fenilpropanolamina $\mathrm{Hcl} / \mathrm{clemastina,} \mathrm{fumarato}$

fenilpropanolamina $\mathrm{Hcl} /$ glicerol iodado

fenilpropanolamina/clorfeniramina/acetaminofén

fenoterol, bromhidrato de

fenoterol/bromhexina

fominobén

formoterol

guaifenesina/terbutalina, sulfato de

hidrocortisona

hidroxizina

ipratropio

ipratropio/fenoterol

ketotifeno

lactato de bamipina/cloruro de amonio/citrato de sodio

loratadina

loratadina/sulfato de pseudoefedrina

metaproterenol

metilprednisolona

montelukast sódico

\section{(Continúa)}


A nexo

(Continuación)

morclofone

nedocromilo sódico

noscapina/glicerilguayacol/efedrina

oximetazolina

paracetamol/cafeína/efetonina

paracetamol/pseudoefedrina

parametasona/clorfeniramina

prednisolona, fosfato sódico sol.

prednisona

\section{pseudoefedrina}

pseudoefedrina/azatadina

pseudoefedrina/bromfeniramina

pseudoefedrina/carbinoxamina

pseudoefedrina/naproxén

salbutamol

salbutamol/beclometasona, dipropionato

salmeterol

teofilina

terbutalina

terfenadina

terfenadina/pseudoefedrina

triamcinolona

triprolidina/pseudoefedrina

tulobuterol, clorhidrato

14. Vitaminas, hematínicos Y electrolitos aminocaproico, ácido ascórbico, ácido

ca. gluconato/ca. lactato/ca. fumarato/sodio, arsinato/lisina/...

calcio, carbonato calcio, carbonato de/colecalciferol

calcio, carbonato de/calcio, glicerofosfato/ complejo B

calcio, caseinato de

calcio/acido ascórbico

calcitriol

cianocobalamina/tiamina

cianocobalamina/tiamina/vit. B6/dexametasona

complejo B

complejo B/lidocaína

complejo B/lisina

factor IX humano

factor VIII humano

ferroso, fumarato

ferroso, fumarato/tiamina

ferroso, sulfato

fitomenadiona

fitomenadiona/carbazocroma

fólico, ácido

folínico, ácido

hidroxocobalamina

hierro dextrán

hierro/cianocobalamina/lisina

menadiona

metenolona

multivitamínicos

oseínico mineral, complejo

piridoxina

potasio, bicarbonato de /KCl/L-lisina/ ácido cítrico

potasio, cloruro de potasio, gluconato de

retinol

retinol/ergocalciferol

sodio, floruro de

soluciones de electrolitos orales

tiamina, clorhidrato de

tiamina, clorhidrato de/piridoxina/cianocobalamina

vitamina A (beta caroteno)

vitamina A palmitato/ergocalciferol

vitamina $B$ y $C$

vitamina $\mathrm{E}$

vitaminas $A, C$ y $D$

15. Misceláneos

acetilcisteína

alprostadil

antiveneno polivalente, crotálico

antiveneno, centuroide escorpión

carnitina

flavoxato

floroglucinol/trimetilfloroglucinol

gestonorona

lípidos intravenosos

nalidíxico, ácido/fenazopiridina

nicotina

pipemídico, ácido

sabal serrulata/utica-dioica, extracto de

seronea repens, extracto de

\section{INGREDIENTES ACTIVOS ESENCIALES EXCLUIDOS}

acexámico, ácido/neomicina

amantadina/clorfeniramina/fenilpropanolami-

na/paracetamol

amantadina/fenilefrina/clorfeniramina/acetilsa-

licílico ác./paracetamol

ambufilina/fenilefrina/etafedrina/doxilamina

amino filina/efedrina/atro pina/fenobarbital

ampicilina/carbocisteína

ampicilina/dicloxacilina

anfepramona

antígenos (Staphyloccus sp./Streptococcus sp./

Neisseria c.)

antígenos de lisado bacteriano

antígenos multibacterianos lisados

asparagina/glutamina/vitamina B6/fosforilserina

betametasona, valerianato/dipropionato

betametasona/dexclorfeniramina

betametasona/gentamicina

betametasona/yodohidroxiquinoleína

bioflavonoides/ácido ascórbico

bumadizona cálcica

caolín y pectina (en combinación) cefaloridina

centella asiática/metronidazol/nitrofurazona

clobetazol

clofibrato

clorfenamina/fenilpropanolamina/paracetamol/cafeína

clorfenamina/metoxifenamina/cafeína/ácido acetilsalicílico

clorfenamina/salicilamida

cloroquinol/hidrocortisona

clorzoxazona/paracetamol/metocarbamol/ ASA

clotrimazol/acetato de dexametasona

colistín/furazolidona/diyohidroxiquinoleína/ diciclomina

dexametasona/neomicina/polimixina B/fenilefrina

dextrometorfano/clorfenamina/salicilato de sodio/cafeína

dextrometorfano/clorfenamina/sulfo guayacol/ fluído de ipeca dextrometorfano/guayacol/metamizol/alcanfor/eucalipto

dextrometorfano/pseudoefedrina/paraceta$\mathrm{mol} / \mathrm{clorfeniramina}$

diflucortolona/clorquinaldol

dipiridamol/ácido acetilsalicílico

disulfirano

efedrina/bálsamo de tolú

eritromicina, estolato

eritromicina/bromhexina

estreptocinasa/estreptodornasa

eto fibrato

eucalipto/guayacol/codeína/belladona/acónito

fenformina

fenilbutazona

fenilefrina/fenildiamina/paracetamol/cafeína fenilefrina/paracetamol/clorfeniramina/cafeína fenipentol/benoctidín/tiamina/nicotinamida fluocinolona

fluocinolona/nistatina/metronidazol

fluocinolona/clioquinol

fluocinolona/neomicina

\section{(Continúa)}




\section{Anexo}

\section{(Continuación)}

fluocinonida

furazolidona/homatropina/peptina

gentamicina/dexametasona

glibenclamida/fenformida

glucomanano (polvo de kojac)

gonadotro pina coriónica/tiamina/hidroxicobalamina

guaifenesina/paracetamol/fenilefrina/clorfenamina

halcinonida

halcinónido/nistatina/sulfato de neomicina hexetidina

isoprenalina/ambufilina/bromhexina isoprinosina (metiso prinol inosina pranobex) lactobacilos acidófilos

lactobacilos/complejo B/factor antitóxico del hígado

loratadina/sulfato de pseudoefredrina mebendazol/niclosamida/tinidazol mentol/alcanfor/eucalipto/trementina metamizol/clormezanona

metformina/clorpropamida

metronidazol/nistatina

neomicina/furazolidona/caolín/pectina/homatropina

neomicina/polimixina B/fluocinolona

nifuroxazida

oxatomida

paracetamol/piricamina/fenilefrina/clorfeniramina paracetamol/pseudoefedrina/dextrometorfano/doxilamina

pipazetato

pipenzolato, bromuro de

pipenzolato, bromuro de/dimeticona

piracetam

polimixina B/neomicina/dexametasona

polimixina B/neomicina/hidrocortisona

polimixina $\mathrm{B} /$ triamcinolona

prifinio, bromuro de

resorcinol/azufre/triclosán rifampicina/trimetoprim

saccharomices boulardii

serratio peptidasa

sulfametoxazol/trimeto prim/guaifenesina/ cloruro de amonio

sulpiride/diacepam

terizidona/fenazopiridina

terpenos, asociación de (mentol, mentona, pineno, a. de oliva)

tetraciclina/guaifenesina/canfusulfonato de calcio/terpina

tianfenicol

tolciclato

tolnaftato

undecilénico, ácido/undecilenato de zinc/ ácido propiónico

vincamida

vincamida/ácido ascórbico

zipeprol 